\title{
Role of magnetic resonance imaging in pregnancy-associated obstetric and gynecological complications
}

\author{
Gayathri Nagenthran ${ }^{1 *}$ (D) Rajeshwaran Rangasami², Anupama Chandrasekharan ${ }^{2}$, Poovini Soundararajan $^{2}$ and \\ Usha Rani Godla²
}

\begin{abstract}
Background: To study the role of MRI in diagnosing pregnancy-associated obstetric and gynecological complications. We prospectively studied 48 pregnant and postpartum women, aged between 20 and 45 years, who were referred for MRI between August 2016 and April 2018 with suspected pregnancy-associated obstetric and gynecological complication. MRI findings were confirmed by intra-operative or histological findings.

Results: Out of the 48 patients, there were 32 women with suspected placenta accreta, 5 pregnant women with 6 ectopic pregnancies ( 1 patient had two gestational sacs), 3 with ovarian pathologies, 3 with uterine pathologies, 3 with abruptio placenta, and 2 with miscellaneous pathologies. In our study, there was an accuracy of $81.6 \%$ with sensitivity of $100 \%$ and specificity of $50 \%$. There was $100 \%$ accuracy in predicting abruptio placenta, ovarian, uterine, and miscellaneous pathologies by MRI. There was $83.4 \%$ and $75 \%$ accuracy in predicting ectopic pregnancy and placenta accreta, respectively, by MRI.
\end{abstract}

Conclusion: MRI is very useful to diagnose pregnancy-associated obstetric and gynecological complications. It will be an important complementary tool to sonography and thereby facilitate better patient management.

Keywords: Pregnancy, Ectopic, Uterine pathology, Ovarian complication

\section{Background}

Ultrasound (USG) is the modality of choice in imaging pregnant patients with abdominal and pelvic pathologies. In advanced pregnancy due to poor acoustic window, there is difficulty in evaluating posterior placenta and adnexal regions. Small field of view (FOV) in sonography makes evaluation of congenital uterine anomalies difficult [1]. USG cannot differentiate hemorrhage from fluids. This is important in diagnosing antepartum hemorrhage and ectopic pregnancies. MRI is safe in pregnancy, has advantages like large FOV coverage, high soft tissue contrast, differentiate blood from fluid, and characterize lesions based on T1/T2 properties [1-3]. Hence, MRI is being increasingly used in patients, where the sonographic diagnosis is ambiguous [4-6]. We studied the role of MRI in diagnosing pregnancy-associated

\footnotetext{
* Correspondence: g3nagikrish@gmail.com

${ }^{1}$ Medall Diagnostic Service Center, Tirunelveli, India

Full list of author information is available at the end of the article
}

obstetric and gynecological complications as there are scanty data available.

\section{Methods}

Institutional ethics committee approval was obtained. We prospectively studied 48 women (46 pregnant and 2 postpartum women), aged between 20 and 45 years (mean 32.5 years), who were referred for MRI between May 2014 and April 2017 with suspected pregnancyassociated obstetric and gynecological complications based on prenatal sonography or inconclusive sonogram or high-risk patients. MRI findings were correlated with final diagnosis arrived after intra-operative as well as histological findings. We excluded patients, who were lost for follow-up. The MRI scans were performed on a $1.5 \mathrm{~T}$ Siemens Magnetom Avanto (Erlangen) or GE 1.5 T Signa HDX (Milwaukee) scanner. A16-element phased-array torso coil was used. In early pregnancy and postpartum cases, since the FOV 
was limited to the pelvis, high-resolution turbo spin echo (TSE) T2-weighted images were obtained in axial, coronal, and sagittal planes with respect to the uterus. Additional T1, T1 fat-suppressed TSE images, short tau inversion recovery (STIR), diffusion-weighted (DWI), and gradient (GRE) images were obtained in axial plane. In 2nd and 3rd trimester pregnancies, to obtain abdominopelvic imaging and to overcome limitations like maternal breathing and fetal movement, the following sequences were used: (i) T2 sections in axial, coronal, and sagittal planes were obtained with half-Fourier acquisition single-shot turbo spin echo (HASTE) and (ii) true fast imaging with steady state precession (TruFi) with fat suppression (FS). T1 and T1 fat-suppressed axial images were obtained with fast spoiled gradient sequence (FSPGR). DWI and echo planar imaging (EPI) gradient (GRE) images in axial planes were also obtained. Dynamic contrast MRI was performed in 2 post partum women in the evaluation of placenta accreta and in 1 woman with gestational trophoblastic disease. The whole body-specific absorption rate was maintained within permissible limits.

\section{Results}

Out of the 48 patients who were referred for MRI, 5 patients were diagnosed with ectopic pregnancy, 3 patients with abruptio placenta, 3 patients with uterine pathologies, 3 patients with ovarian pathologies, and 2 with miscellaneous pathologies. There were 32 patients where placenta accreta was suspected (Table 1). The sites of ectopic pregnancy in 5 patients included three interstitial pregnancy (two sacs were seen in 1 patient), 1 angular pregnancy, 1 scar pregnancy, and 1 twin pregnancy with second twin in the left tube of the uterus. Three uterine pathologies we came across were 2 cases of uterine fibroids with red degeneration and 1 case of uterine dehiscence. The 3 ovarian pathologies encountered were ovarian torsion, and the two miscellaneous cases were hydrop degeneration in missed abortion and invasive mole. There was $100 \%$ correlation between MR imaging findings and the final outcome in cases with abruptio placenta, ovarian, uterine, and miscellaneous pathologies. There was $83.4 \%$ and $75 \%$ accuracy in predicting ectopic pregnancy and placenta accreta, respectively, by MRI. There was an accuracy of $81.6 \%$ with sensitivity of

Table 1 Showing the MRI findings in the 48 patients and the final diagnosis as confirmed by surgery/histopathology

\begin{tabular}{|c|c|c|c|}
\hline MRI findings & No. of lesions & Final diagnosis & $\begin{array}{l}\text { MRI and final diagnosis } \\
\text { correlation }\end{array}$ \\
\hline Ectopic pregnancy & 6 & & \\
\hline Interstitial pregnancy & 3 & Interstitial pregnancy & Positive \\
\hline Scar pregnancy & 1 & Scar pregnancy & Positive \\
\hline $\begin{array}{l}1 \text { fetus in right cornu and other in } \\
\text { rudimentary left horn }\end{array}$ & 1 & $\begin{array}{l}\text { Left tubal pregnancy, this fetus } \\
\text { died later in the pregnancy }\end{array}$ & Negative \\
\hline Left angular pregnancy & 1 & Left angular pregnancy & Positive \\
\hline Suspected placenta accreta & 32 & & \\
\hline Placenta accreta-positive & 15 & Placenta accreta-positive & Positive \\
\hline Placenta accreta-positive & 8 & Placenta accreta-negative & Negative \\
\hline Placenta accreta-negative & 9 & Placenta accreta-negative & Positive \\
\hline Abruptio placenta & 3 & & \\
\hline Partial abruption & 2 & Partial abruption & Positive \\
\hline Preplacental abruption & 1 & Preplacental abruption & Positive \\
\hline Uterine pathologies & 3 & & \\
\hline Red degeneration of uterine fibroid & 2 & Red degeneration of uterine fibroid & Positive \\
\hline Uterine dehiscence & 1 & Uterine dehiscence & Positive \\
\hline Ovarian pathologies & 3 & & \\
\hline Ovarian torsion & 1 & Ovarian torsion & Positive \\
\hline Ovarian cyst torsion & 1 & Ovarian cyst torsion & Positive \\
\hline Ovarian mass torsion & 1 & Immature teratoma torsion & Positive \\
\hline Miscellaneous pathologies & 2 & & \\
\hline Hydropic degeneration & 1 & Hydropic degeneration & Positive \\
\hline Invasive mole & 1 & Invasive mole & Positive \\
\hline
\end{tabular}


$100 \%$ and specificity of $50 \%$ in diagnosing pregnancyassociated obstetric and gynecological complications.

\section{Discussion}

\section{Ectopic pregnancy (EP)}

Ectopic pregnancy refers to implantation of a fertilized ovum outside of the uterine cavity. The commonest site for ectopic pregnancy is ampulla of the fallopian tube. Other sites include ovaries, peritoneal cavity, omentum, and intra-uterine sites like uterine interstitial, cervix, cesarean scar, and anomalous uterine horn [5]. Imaging features include empty uterine cavity with no intrauterine gestational sac (GS) and thick echogenic endometrium. Other extra-uterine findings include extra-uterine gestational sac with embryo and yolk sac within, solid mass is also possible, free fluid, or hemoperitoneum in the pelvis. Advantage of MRI over TVS is identification of blood and identifying unusual presentation of extra tubal pregnancies. Hallmark of GS is blood within its walls, and it appears as cystic structure with low signal intensity in its wall on T2WI, suggestive of hemorrhage [1].. Mimicking conditions such as corpus luteal cyst do not contain hemorrhage. In interstitial pregnancy, GS is seen in interstitial segment of the fallopian tube, i.e., in the lateral angle of uterine fundus and is surrounded by $<5 \mathrm{~mm}$ of myometrium. These findings are well depicted on MRI as in our case. It has to be differentiated from angular pregnancy as the former is associated with hemorrhage and rupture [5]. Angular pregnancy refers to implantation within the endometrium of lateral angle of the uterus, medial to the utero tubal junction. These pregnancies can be observed as the gestational sac may later reach the uterine cavity. In scar pregnancy, the fertilized ovum implants over the fibrous scar of previous cesarean section or other uterine surgeries. In our case, MRI showed thinned out myometrium between gestational sac and urinary bladder and helped to differentiate from a miscarriage in progress.

In our study, there were 5 patients with 6 ectopic pregnancies and MRI was performed to obtain additional information about localization of gestational site. MRI was useful in making a correct diagnosis in 5 pregnancies (accuracy of $83.3 \%)$. In two patients with 3 interstitial pregnancies (1 patient had 2 sacs), there was a doubt on USG regarding interstitial/angular pregnancy; MRI could confirm the diagnosis of interstitial pregnancy. MRI could demonstrate uterine wall medial to the gestational sac suggesting interstitial pregnancy (Fig. 1). In another patient where scar pregnancy was initially suspected on USG, MRI could show the gestational sac and the thinned out anterior myometrium. In one case of intra-uterine and tubal pregnancy (twins) at 28 weeks gestational age, the tubal pregnancy was reported on MRI as pregnancy in a rudimentary horn. Laparotomy led to a final diagnosis of tubal pregnancy in this patient. Thus, when there is advanced pregnancy, it may be sometimes difficult to diagnose the exact site of the EP. Yoshigi et al. [6] in their prospective study of 24 patients with suspected ectopic pregnancy concluded that MRI had 95\% sensitivity, 100\% specificity, and 96\% accuracy in diagnosing EP and found T2* images most useful.

\section{Placenta accreta (PA)}

Placenta accreta is a defect in the decidua basalis allowing the invasion of chorionic villi in to the myometrium. Common causes include previous cesarean section, any previous uterine surgery and placenta previa [7]. MRI is complementary when US findings are equivocal and to

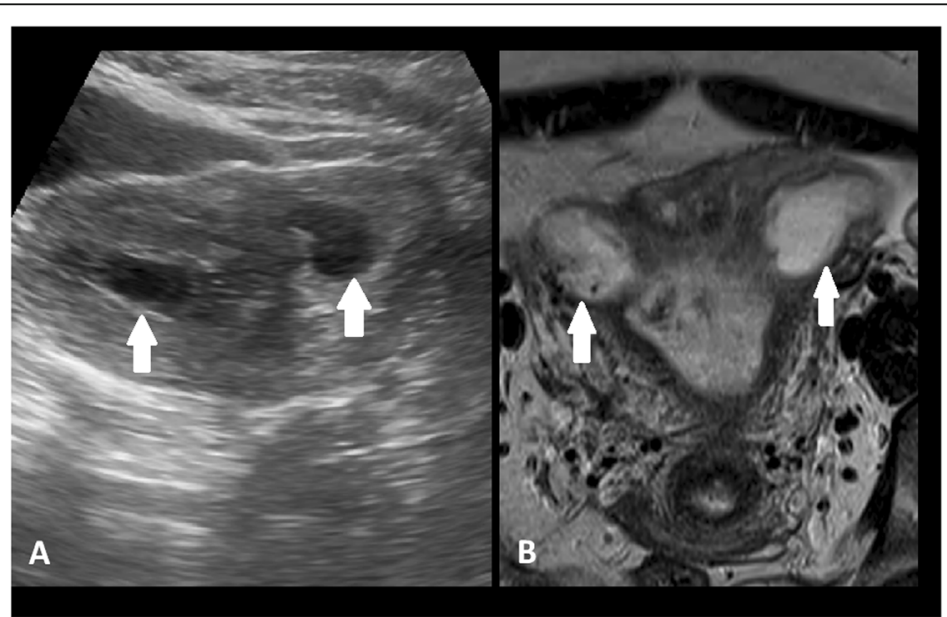

Fig. 1 a USG image showing two gestational sacs, one in each cornu of the uterus, suggesting the presence of interstitial/ angular pregnancy. $\mathbf{b}$ T2-weighted coronal image shows two gestational sacs, one in each cornu (arrow), lateral to the uterotubal junctions suggesting bilateral interstitial pregnancy 
identify the depth of invasion. MRI findings are heterogeneous signal intensity of the placenta due to lacunae or hemorrhage within, bulging of myometrium with loss of normal pear shape of the gravid uterus, nodular or linear low-signal intensity on T2 WI (intra-placental bands), and focal areas of myometrial thinning or interruptions [8]. There is a controversy regarding contrast-enhanced MRI in placenta accreta. Currently, gadolinium [Gd] is used on selected patients who are elected for immediate cesarean section or termination of pregnancy [9].

There were 32 patients suspected to have PA by USG. There were 23 patients diagnosed as PA by MRI. However, on follow-up, only 15 turned out to have placenta accreta. MRI ruled out PA in 9 patients that were subsequently proved on follow-up (Table 1). There was $75 \%$ accuracy in predicting placenta accreta by MRI. MR criteria for diagnosis PA where thinning of the myometrium $[<1 \mathrm{~mm}]$, loss of placental endometrial interface, focal uterine bulge, multiple T2 dark bands, and heterogeneous placenta (Fig. 2). There were no false negatives. MRI over diagnosed PA in 8 cases. Thinning of myometrium or loss of interface was seen in these false positive cases due to advanced pregnancy. Volume averaging and

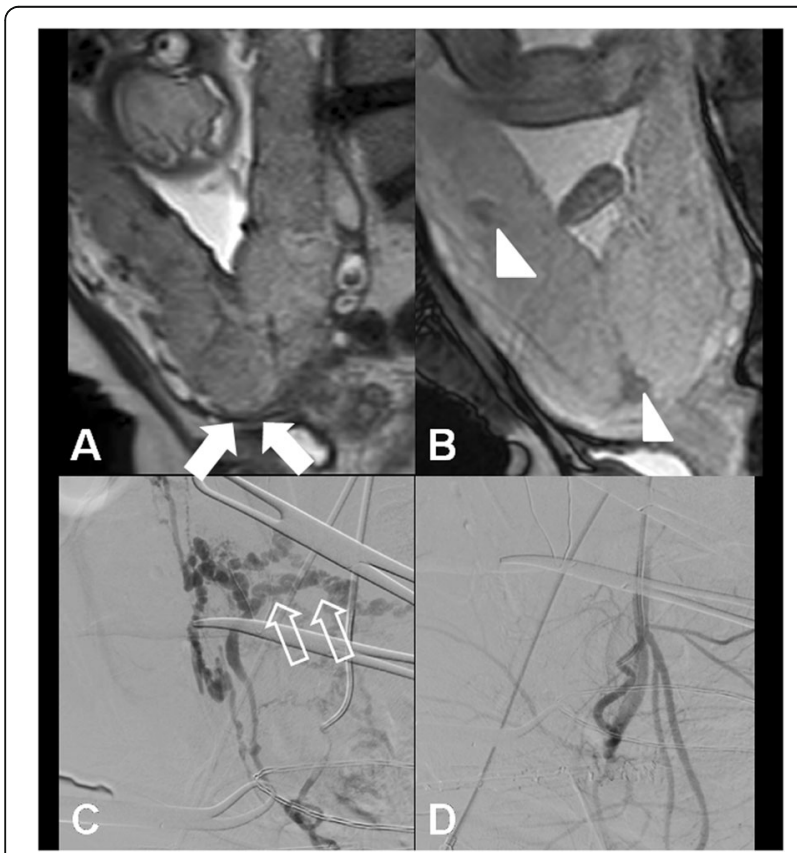

Fig. 2 A 33-week pregnant female with suspected placenta accreta on USG. a MR imaging T2W sagittal image shows type 4 placenta previa with loss of myometrial interface (arrow) confirming placenta accreta. b T2WI shows dark bands (arrow heads). c Pre-embolization angiogram shows hypervascularity arising from the uterine artery (open arrows). b Post embolization uterine angiogram shows reduction in hypervascularity. The fetus was delivered by LSCS, and the placenta was left in situ that got expelled later on respiratory movements also caused erroneous interpretation of loss of interface. Various authors have found MRI useful in PA with accuracy reaching around $70 \%$. Riteau et al. [4] studied 42 patients with suspected placenta accreta and concluded that USG showed a sensitivity and specificity of $100 \%$ and $37.5 \%$ in diagnosis of PA whereas MRI showed a sensitivity and specificity of $76.9 \%$ and $50 \%$.

\section{Abruptio placenta (AP)}

USG is the primary modality in identifying abruption placenta, and MRI gives a better resolution and diagnoses even in USG negative cases. Imaging findings include hematoma formation which can be retroplacental, subchorionic, and pre-placental. It can be further classified as hyperacute, acute, subacute, and chronic [10]. Due to anechoic nature of the acute hemorrhage, it is sometimes difficult to diagnose only with USG. MRI is helpful to diagnose abruption and the age of the blood clot. MRI is recommended whenever the patient is stable.

There were 3 cases of AP and were diagnosed correctly by MRI (100\% accuracy).This included 2 cases of retroplacental hematoma and one with the preplacental hemorrhage. This correlates with study done by Masselli et al. [10] who found $100 \%$ accuracy with MRI in diagnosing AP and only $52 \%$ by USG. In one of our patients, the hemorrhage was appearing anechoic on USG (Fig. 3) and was misdiagnosed. However, it was confidently diagnosed on MR examination.

\section{Uterine pathologies}

Uterine rupture is a life-threatening condition which is defined as full-thickness tear of the uterus and involving the visceral peritoneum [1]. USG can detect hemoperitoneum. However, predicting actual site of disruption may be difficult by USG. MRI with its better spatial resolution can detect the actual site of uterine disruption. However, MRI is recommended only when the patient is stable.

There were 2 cases of red degeneration in uterine fibroid and 1 case of uterine rupture (Table 1). All these conditions could be diagnosed by MRI accurately. Helpful feature to diagnose the degeneration was the presence of hemorrhagic products in the fibroid (Fig. 4). The site of uterine rupture was precisely diagnosed by MRI whereas it could only be suspected on ultrasound (Fig. 5). Red degeneration of fibroid is most common in pregnancy secondary to hemorrhagic infarction due to obstructed draining veins and may be demonstrated by MRI $[11,12]$.

\section{Ovarian pathologies}

There were 3 cases of ovarian torsion and were diagnosed correctly by MRI. The differential diagnosis is hemorrhagic cyst and ovarian hyperstimulation syndrome. MRI features of ovarian torsion include enlarged ovaries with stromal 


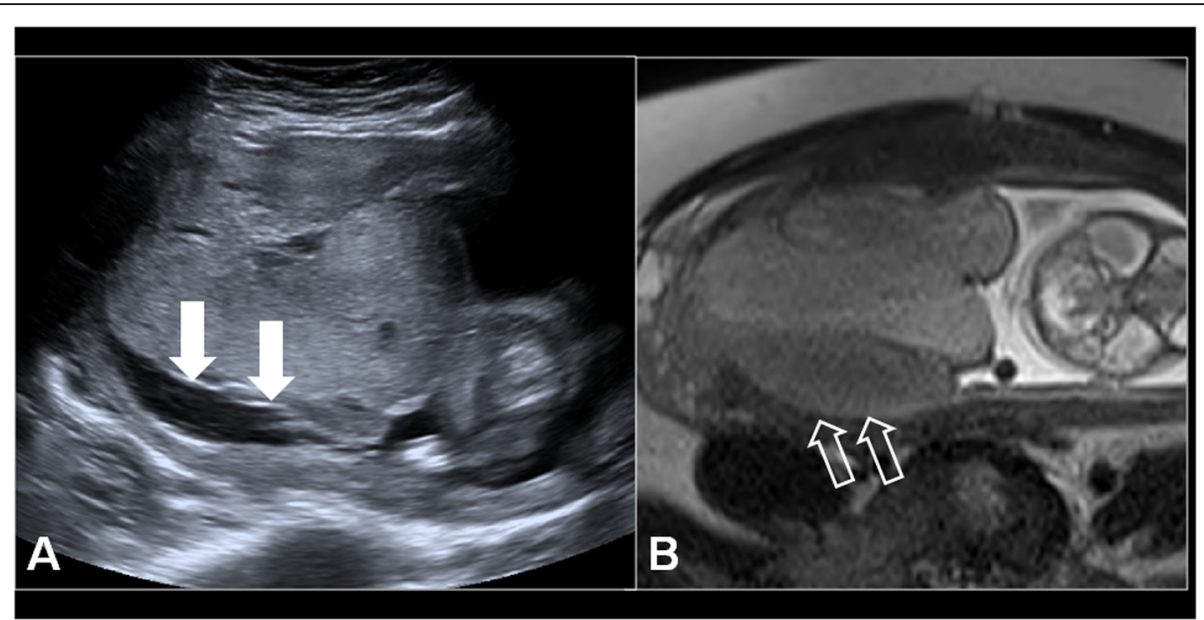

Fig. 3 A 21-week pregnant female with bleeding PV. a USG image shows displacement of the placenta from the posterior uterine wall (arrows). Hemorrhage could not be made out. b T2 WI shows sub-acute retroplacental clot in the posterior aspect (open arrows) suggesting abruptio placenta. Retrospectively, the hemorrhage was seen as anechoic area on USG

edema/hemorrhage (Fig. 6), twisting and thickening of pedicle of $>10 \mathrm{~mm}$ [1], free fluid in the pelvis, and absence of post gadolinium enhancement [1].

\section{Miscellaneous pathologies}

Miscellaneous pathologies include molar pregnancy, choriocarcinoma, and hydropic degeneration. Molar pregnancy can be further classified into partial and complete mole. Though USG is the modality of choice in molar pregnancy, invasion of the uterine wall is better seen on MRI in invasive mole than on
USG. Post contrast administration of gadolinium will show avid enhancement of the lesion. MRI is superior to USG in identification of extrauterine extensions [13]. MRI in molar pregnancy is limited to obese patients and co-existing other pathologies like leiomyoma. Imaging findings include high-signal intensity on T2WI and low-signal intensity in T1WI with a sharp border between the myometrium and the mass. Ultrasound appearance in failing pregnancy with hydropic degeneration is numerous cystic spaces with placental enlargement. It can be differentiated from

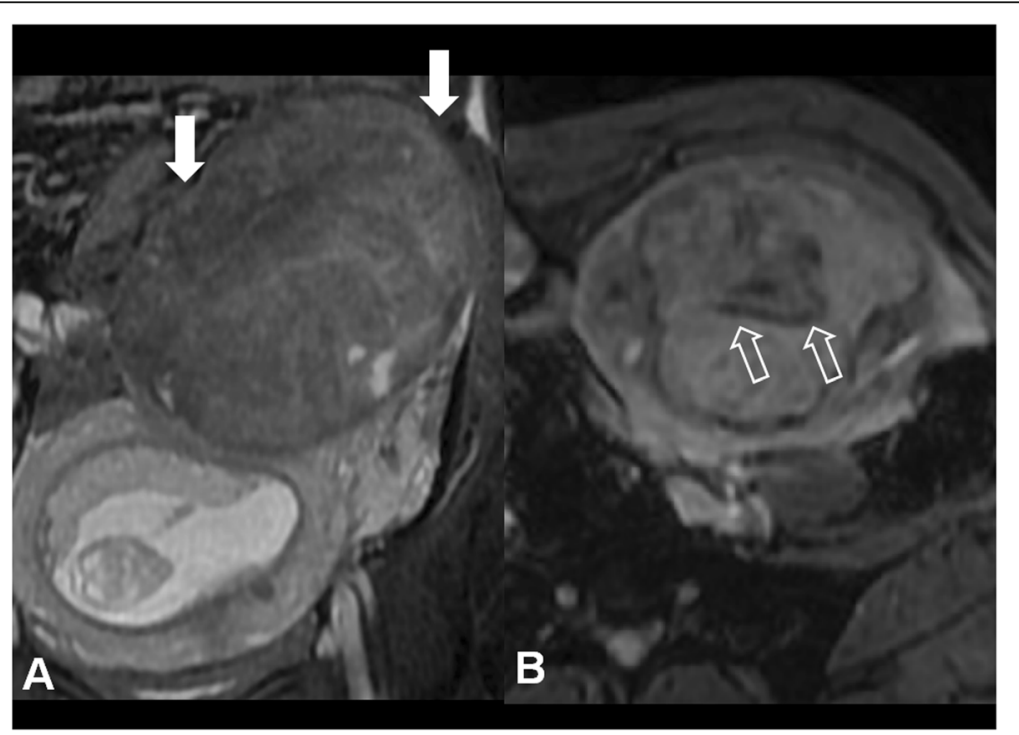

Fig. 4 A 31-year-old pregnant patient with abdominal pain. a Coronal T2W image shows a heterogeneous sub-serosal fibroid in the fundus (arrows). b Axial gradient image (GRE) shows areas of hemorrhage within (open arrows) suggesting red degeneration. The patient was treated conservatively and myomectomy performed after delivery 


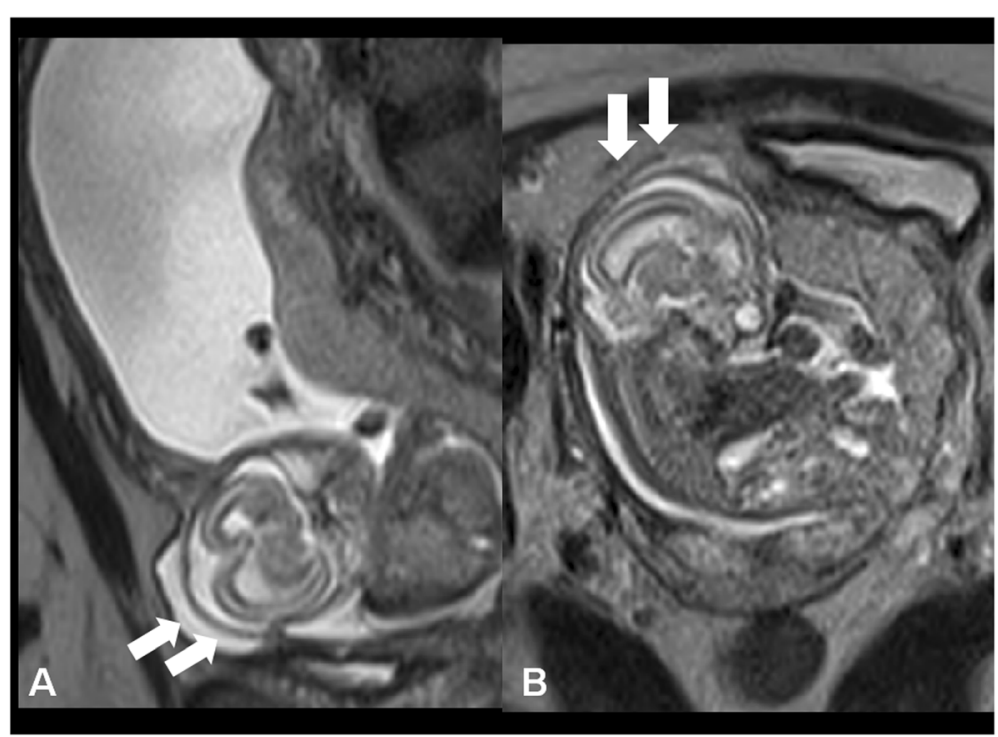

Fig. 5 a Sagittal T2 W. b Axial T2W images show myometrial thinning with focal bulge in the right lateral aspect of the anterior wall of the uterus (arrows), in the region of the previous cesarean scar suggesting uterine dehiscence

the partial mole by the normal-sized uterus and low/ declining beta HCG. MRI has no significant role in differentiating both, and histopathological diagnosis is confirmatory in this condition [14].

There were 2 miscellaneous pathologies: one was hydrop degeneration in missed abortion and the other was invasive mole (Table 1). Both were correctly diagnosed by MRI. Invasion of the uterine wall was well seen on MRI in invasive mole.

\section{Conclusion}

USG is the primary tool in the evaluation of pregnancyassociated obstetric and gynecological complications. MRI is a complementary tool in view of its high softtissue resolution and ability to characterize tissues based on application of various sequences. MRI aids in diagnosing pathologies where USG findings are equivocal and help in planning further management, thus preventing maternal morbidity and mortality.

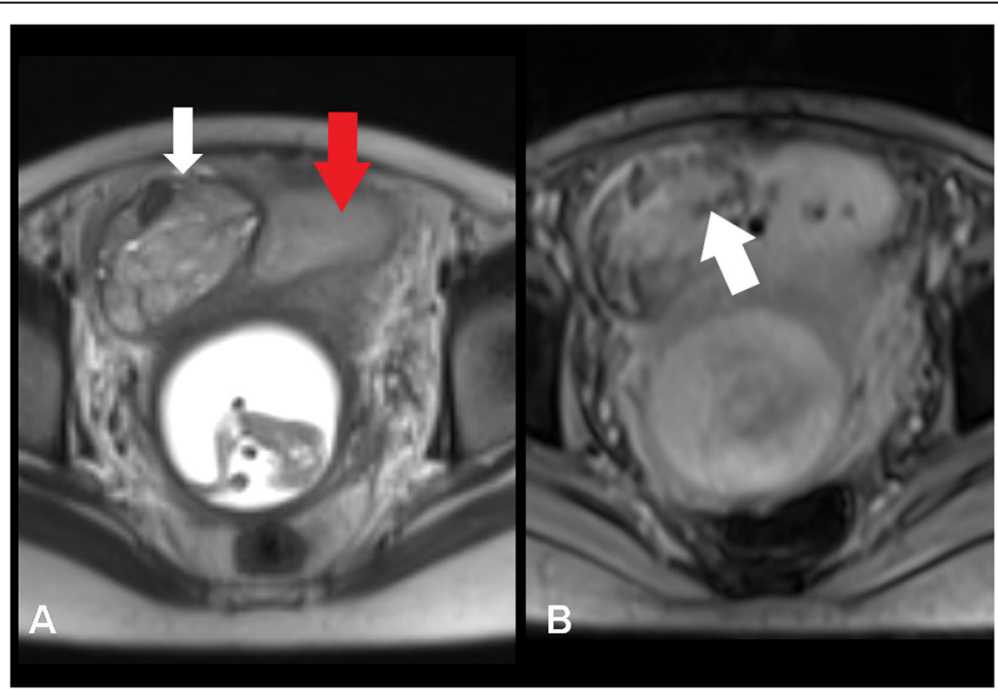

Fig. 6 Pregnant female with right iliac fossa pain. a Axial T2 W image shows enlarged right ovary with peripherally placed follicles(white arrow) and adjacent fluid (red arrows). b Axial gradient (GRE) image shows hemorrhage within suggesting ovarian torsion (white arrow) 


\section{Abbreviations}

DWl: Diffusion-weighted imaging; EP: Ectopic pregnancy; FOV: Field of view GS: Gestational sac; MRI: Magnetic resonance imaging; PA: Placenta accreta; TVS: Transvaginal ultrasound; USG: Ultrasonography

\section{Acknowledgements}

Not applicable.

\section{Authors' contributions}

GN contributed to the design, definition of intellectual content, literature search, clinical studies, experimental studies, data analysis, statistical analysis, manuscript preparation, manuscript editing, manuscript review, and guarantor of the study. RR contributed to the concepts, design, definition of intellectual content, literature search, data acquisition, data analysis, statistical analysis, manuscript editing, manuscript review, and guarantor of the study. AC contributed to the concepts, design, literature search, clinical studies, experimental studies, data analysis, and manuscript review. PS contributed to the concepts, design, definition of intellectual content, clinical studies, experimental studies, data acquisition, data analysis, and manuscript review. UG contributed to the concepts, definition of intellectual content, clinical studies, experimental studies, clinical studies, experimental studies, data acquisition, data analysis, and manuscript review. All authors have read and approved the manuscript and ensure that this is the case.

\section{Funding}

This study had no funding from any resource.

\section{Availability of data and materials}

The datasets used and/or analyzed during the current study are available from the corresponding author on reasonable request.

\section{Ethics approval and consent to participate}

This study was approved by the Research Ethics Committee of the Faculty of Medicine at Sri Ramachandra University on August 23, 2016, reference number of approval: CSP-med/15/aug/24/24. All patients included in this study gave written informed consent to participate in this research. If the patient was less than 16 years old or unconscious at the time of the study, written informed consent for their participation was given by their parent or legal guardian.

\section{Consent for publication}

All patients included in this research gave written informed consent to publish the data contained within this study. If the patient was less than 16 years old, deceased, or unconscious when consent for publication was requested, written informed consent for the publication of this data was given by their parent or legal guardian.

\section{Competing interests}

The authors declare that they have no competing interests.

\section{Author details}

${ }^{1}$ Medall Diagnostic Service Center, Tirunelveli, India. ${ }^{2}$ Sri Ramachandra Institute of higher education and research, Chennai, Tamilnadu, India.

Received: 3 October 2019 Accepted: 9 December 2019

Published online: 20 December 2019

\section{References}

1. Gupta R, Bajaj SK, Kumar N et al (2016) Magnetic resonance imaging - a troubleshooter in obstetric emergencies: a pictorial review. Indian J Radiol Imaging 26:44-51

2. Levine D (2001) Ultrasound versus magnetic resonance imaging in fetal evaluation. Topics in Magnetic resonance imaging 12:25-38

3. Rajeswaran R, Chandrasekharan A, Joseph S, Venkata Sai P, Dev B, Reddy S (2009) Ultrasound versus MRI in the diagnosis of fetal head and trunk anomalies. J Matern Fetal Neonatal Med 22:115-123

4. Riteau A, Tassin M, Chambon G et al (2014) Accuracy of ultrasonography and magnetic resonance imaging in the diagnosis of placenta accreta. PLoS One 9:e94866
5. Priya M, Rajeswaran R, Anupama C, Chitra A (2017) Role of MRI in the localisation of uncommon forms of ectopic pregnancies. Int J Contemp Med Res 4:1898-1901

6. Yoshigi J, Yashiro N, Kinoshita T (2006) Diagnosis of ectopic pregnancy with MRI: efficacy of T2 weighted imaging. Magn Reson Med Sci 5:25-32

7. Christopher Baughman W, Corteville JE, Shah RR (2008) Placenta accreta: spectrum of US and MR imaging findings. Radiographics 28:1905-1916

8. Varghese B, Singh N, George RAN et al (2013) Magnetic resonance imaging of placenta accreta. Indian J Radiollmag 23:379-385

9. Millischer AE, Deloison B, Silvera S, Ville Y, Boddaert N, Balvay D et al (2017) Dynamic contrast enhanced MRI of the placenta: a tool for prenatal diagnosis of placenta accreta? Placenta. 53:40-47 pmid:28487019

10. Masselli G, Brunelli R, Di Tola M et al (2011) MR imaging in the evaluation of placental abruption: correlation with sonographic findings. Radiology 259:222-230

11. Radhika BH, Naik K, Shreelatha S, Vana H (2015) Case series: pregnancy outcome in patients with uterine fibroids. J Clin Diagn Res 9:QR01-QR04

12. Wilde S, Scott-Barrett S (2009) Radiological appearances of uterine fibroids. Indian J Radiol Imaging 19:222-231

13. Shaaban AM, Rezvani M, Haroun RR et al (2017) Gestational trophoblastic disease: clinical and imaging features. Radiographics 37:681-700

14. Kathpalia SK, Jayshree Kulkarni, Divya Punetha and Aparna Karan (2018) Hydropic degeneration of placenta - an unusual clinical presentation. Scientia Ricerca Volume .Issue 2: 239 to 241

\section{Publisher's Note}

Springer Nature remains neutral with regard to jurisdictional claims in published maps and institutional affiliations.

\section{Submit your manuscript to a SpringerOpen ${ }^{\circ}$ journal and benefit from:}

- Convenient online submission

- Rigorous peer review

- Open access: articles freely available online

High visibility within the field

- Retaining the copyright to your article

Submit your next manuscript at $\boldsymbol{\nabla}$ springeropen.com 